\title{
Treatment of Refractory Melasma with Microwave-generated, Atmospheric-pressure, Non-thermal Nitrogen Plasma
}

\author{
Hyun-Jo Kim ${ }^{1,2}$ \\ Heesu Kim ${ }^{1}$ \\ Young Koo Kim ${ }^{3}$ \\ Sung Bin $\mathrm{Cho}^{1,4}$
}

\footnotetext{
${ }^{1}$ Department of Dermatology and Cutaneous Biology Research Center, International St. Mary's Hospital, Catholic Kwandong University College of Medicine, Incheon, Korea

${ }^{2}$ CNP Skin Clinic, Cheonan, Korea

${ }^{3}$ Yonsei Star Skin \& Laser Clinic, Seoul, Korea

${ }^{4}$ Yonsei Seran Dermatology and Laser Clinic,

Seoul, Korea
}

\begin{abstract}
Periorbital melasma is often refractory to treatment and highly associated with rebound hyperpigmentation or mottled hypopigmentation after laser treatment in Asian patients. In this report, we describe 2 patients with cluster-1 periorbital melasma and 1 patient with cluster-2 periorbital melasma who experienced remarkable clinical improvements after microwave-generated, atmospheric-pressure, non-thermal nitrogen plasma treatments. All patients exhibited limited clinical responses after combination treatments with topical bleaching agents, systemic oral tranexamic acid, and low-fluenced Q-switched neodymium (Nd):yttrium-aluminum-garnet (YAG) lasers. Low-energy nitrogen plasma treatment at $0.75 \mathrm{~J}$ elicited remarkable clinical improvement in the periorbital melasma lesions without post-laser therapy rebound hyperpigmentation and mottled hypopigmentation. We deemed that a single pass of nitrogen plasma treatment at $0.75 \mathrm{~J}$ induces mild microscopic thermal tissue coagulation and modification within the epidermis while preserving the integrity of the basement membrane in patients with periorbital melasma. Accordingly, nitrogen plasma-induced dermal tissue regeneration could play a role in the treatment of melasma lesions.
\end{abstract}

\section{Key words}

Plasma; Nitrogen; Melasma; Atmospheric-pressure plasma; Nonthermal plasma
Received December 11, 2019

Accepted December 18, 2019

\footnotetext{
Correspondence

Sung Bin Cho

Department of Dermatology and Cutaneous

Biology Research Center, International St. Mary's Hospital, Catholic Kwandong University College of Medicine, 25 Simgok-ro, Seo-gu, Incheon 22711, Korea

Tel.: +82-32-290-3142

Fax: $+82-32-290-3142$

E-mail: drsbchođggmail.com

(c) Korean Society for Laser Medicine and Surgery

(@) This is an open access article distributed under the terms of the Creative Commons Attribution NonCommercial License (http://creativecommons.org/ licenses/by-nc/4.0) which permits unrestricted noncommercial use, distribution, and reproduction in any medium, provided the original work is properly cited.
} 


\section{INTRODUCTION}

Melasma is a common hyperpigmentation disorder in Asian individuals. ${ }^{1-3}$ Various clinical manifestations thereof have been categorized into centrofacial, malar, mandibular, periorbital, and mixed types according to their patterns of melasma distribution. ${ }^{2-4}$ Among the aforementioned types of melasma, periorbital melasma is commonly found in Asian patients; it is often refractory to treatment and highly associated with the risk of rebound hyperpigmentation or mottled hypopigmentation after laser treatment. $^{3}$

A previous hierarchical cluster analysis study identified two statistically different clusters of Asian patients with periorbital melasma. ${ }^{3}$ Therein, patients in cluster 1 were found to have fine and homogeneous melasma pigmentation lesions along the upper and lower eyelids, and the pigmentation thereof became darker closer to the inferolateral margin of the periorbital area. ${ }^{3}$ Patients in cluster 2 were older than those in cluster 1 , and melasma lesions in cluster 2 more commonly distributed throughout the periorbital area, the temple, and the medial cheek. ${ }^{3}$

In the present report, we describe 2 patients with cluster-1 periorbital melasma and 1 patient with cluster-2 periorbital melasma who showed remarkable clinical improvements after treatment with microwave-generated, atmospheric-pressure, non-thermal nitrogen plasma. To deliver nitrogen-plasma pulses to melasma pigmentation lesions, a plasma generator (Pladuo ${ }^{\mathrm{TM}}$; Shenb Co., Ltd., Seoul, Korea) was utilized in this study that converted inert nitrogen gas to excited ionized nitrogen gas via 2.45$\mathrm{GHz}$ microwave energy. The inert nitrogen gas was loaded at a preset volume in a nozzle with a 5-mm diameter, and then, microwave-generated nitrogen-plasma pulses at a preset energy and pulse duration were delivered from the distal end of the handpiece into target skin.

\section{CASE REPORT}

\section{Case 1}

A 42-year-old Korean female visited our clinic presenting with coarse, homogeneous, dark-brownish macular lesions on the face. The patient had been treated with topical bleaching agents and neodymium (Nd):yttriumaluminum-garnet (YAG) laser at other clinics. However, the clinical outcomes thereof were unsatisfactory, and the lesions had progressed. At her first visitation at our clinic, the lesions were symmetrically distributed across the temple, upper and lower eyelids, malar areas, and anterior cheeks (Fig. 1A), and a clinical diagnosis of cluster-2 periorbital melasma was made.

After obtaining written informed consent, the patient was treated with systemic tranexamic acid $(1,000 \mathrm{mg})$ for 3 months, two sessions of fractionated ruby laser (Melastar; Asclepion Laser Technologies GmBH, Jena, Germanyl treatment, and the 15 sessions of low-fluenced 1,064-nm Nd:YAG laser (RevLite ${ }^{\circledR}$ SI; Cynosure, Westford, MA, USA) treatment. The parameters for the fractionated ruby laser treatment included an Fx mode, $4.5 \mathrm{~J}$, and two passes, and those for low-fluenced Nd:YAG laser treatment included a PTP-on mode, 7.0-mm spot size, a fluence of $3.9 \mathrm{~J} / \mathrm{cm}^{2}$, and a clinical end-point of mild erythema on melasma lesions. While the melasma lesions had improved, results were not entirely satisfactory
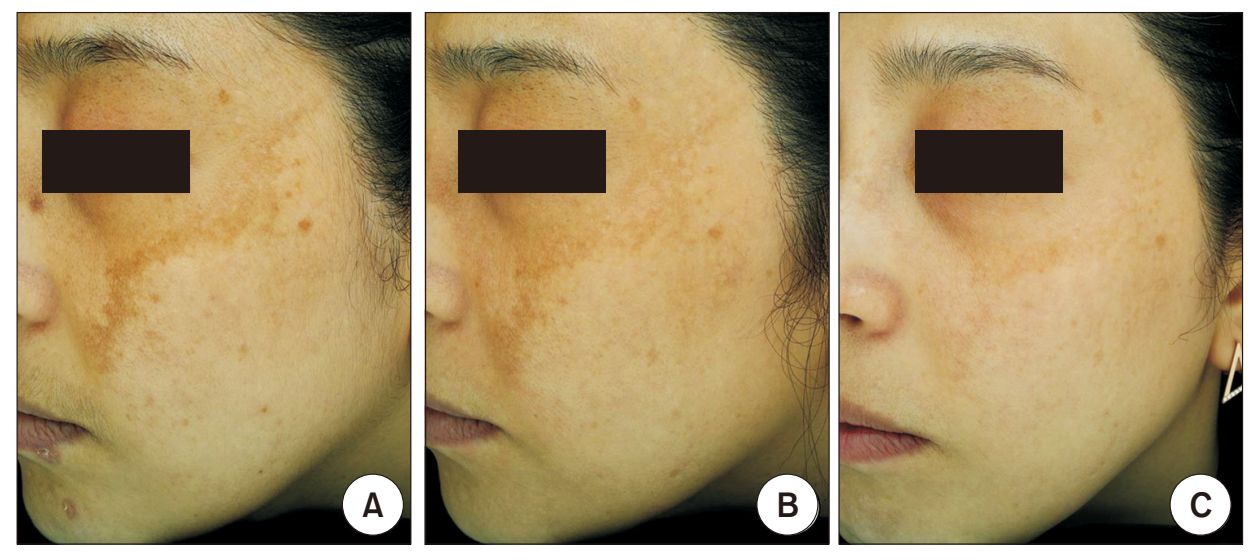

Fig. 1. Case 1. Photographs of a 42-year-old Korean female patient with cluster-2 periorbital melasma (A) at baseline; (B) 1 month after systemic tranexamic acid $(1,000 \mathrm{mg})$ treatment for 3 months, two sessions of fractionated ruby laser treatment, and 15 sessions of low-fluenced 1,064-nm neodymium (Nd):yttrium-aluminum-garnet (YAG) laser treatment; and (C) 1 month after systemic tranexamic acid (1,000 mg) treatment for 3 months, 15 sessions of low-fluenced Nd:YAG laser treatment, and eight sessions of nitrogen plasma treatment. Photographs were taken under normal light exposure. Left lateral view. 
(Fig. 1B). Thus, additional treatment, which comprised treatment with systemic tranexamic acid $(1,000 \mathrm{mg})$ for 3 months, 15 sessions of low-fluenced Nd:YAG laser treatment, and eight sessions of nitrogen plasma treatment, were administered. The treatment settings of the nitrogen plasma treatment comprised a pulse energy of 0.75 $\mathrm{J}$, a pulse duration of $10 \mathrm{msec}$, and a distance from the nozzle's tip to the skin of $10 \mathrm{~mm}$. For combined nitrogen plasma and low-fluenced Nd:YAG laser treatment, after cleansing the patient's face with a mild soap and 70\% ethanol, the entire face was treated with a single pass of nitrogen plasma treatment for a total shot count of approximately 400-500 shots. Immediately thereafter, lowfluenced Nd:YAG laser treatment was performed on the entire face. The treated areas were cooled with icepacks immediately after treatment. No prophylactic systemic or topical corticosteroids, antibiotics, or antiviral agents were prescribed. The patient was recommended to avoid excessive sun exposure.

One month after the last treatment, the patient exhibited marked reductions in melasma pigmentation along the temple, periorbital area, and anterior cheeks (Fig. $1 \mathrm{C})$. Furthermore, her skin texture and overall skin tone were improved, and the patient was satisfied with the results. Pain during the nitrogen plasma and low-fluenced $\mathrm{Nd}$ :YAG laser treatments was tolerable without the use of topical anesthetic cream, and most of the post-treatment erythema spontaneously disappeared within 2 hours. No remarkable major side effects, including itching, crusts, oozing, burn, folliculitis, prolonged edema or erythema, rebound hyperpigmentation, mottled hypopigmentation and scarring, were encountered.

\section{Case 2}

A 40-year-old Korean female visited our clinic presenting with coarse, homogeneous, brownish lesions on the face. The patient had been treated with topical bleaching agents at another clinic, but the results were unsatisfactory. At the first visitation at our clinic, the lesions were symmetrically distributed primarily on the periorbital areas (Fig. 2A), and a clinical diagnosis of cluster-1 periorbital melasma was made.

After obtaining written informed consent, the patient was treated with systemic tranexamic acid $(1,000 \mathrm{mg})$ for 2 months, one session of intense pulsed light (IPL, ICON ${ }^{\mathrm{TM}}$ Max G Handpiece; Cynosurel treatment, and 15 sessions of low-fluenced 1,064-nm Nd:YAG laser (RevLite ${ }^{\circledR}$ SI) treatment. The parameters of IPL treatment included a pulse duration of $15 \mathrm{msec}$, a fluence of $36 \mathrm{~J} / \mathrm{cm}^{2}$, and two to three passes, and those of low-fluenced Nd:YAG laser treatment included a PTP-on mode, a 7.0-mm spot size, a fluence of $3.9 \mathrm{~J} / \mathrm{cm}^{2}$, and a clinical end-point of mild erythema on melasma lesions. The melasma lesions improved, but results were not satisfactory (Fig. 2B). Thus, additional treatment, which comprised treatment with systemic tranexamic acid $(1,000 \mathrm{mg}$ ) for 3 months, 10 sessions of low-fluenced Nd:YAG laser treatment, and eight sessions of nitrogen plasma treatment, were delivered. The nitrogen plasma was prepared at a pulse energy of $0.75 \mathrm{~J}$, a pulse duration of $10 \mathrm{msec}$, and a distance from the nozzle's tip to the skin of $10 \mathrm{~mm}$. Combined nitrogen plasma and low-fluenced Nd:YAG laser treatment and post-treatment care were performed according to methods described above.

One month after the last treatment, the patient exhib-
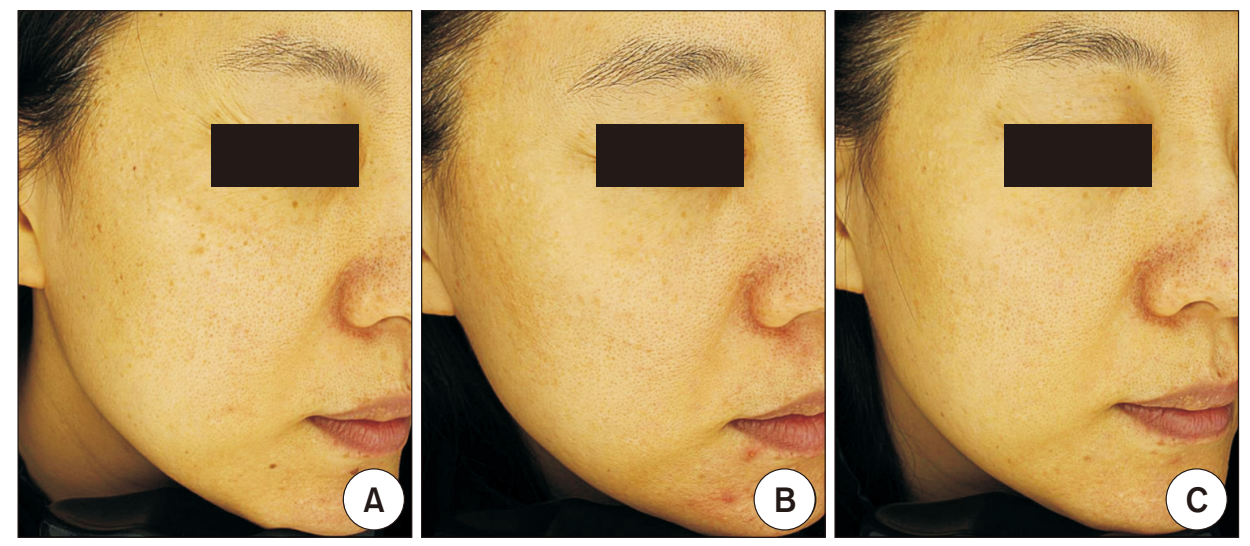

Fig. 2. Case 2. Photographs of a 41-year-old Korean female patient with cluster-2 periorbital melasma (A) at baseline; (B) 1 month after systemic tranexamic acid $(1,000 \mathrm{mg})$ treatment for 2 months, one session of intense pulsed light (IPL) treatment, and 15 sessions of low-fluenced 1,064-nm Nd:YAG laser treatment; and (C) 1 month after systemic tranexamic acid (1,000 mg) treatment for 2 months, 10 sessions of low-fluenced Nd:YAG laser treatment, and eight sessions of nitrogen plasma treatment. Photographs were taken under normal light exposure. Right lateral view. 
ited marked reductions in melasma pigmentation on the temple, periorbital area, and anterior cheeks (Fig. 2C). Furthermore, her skin texture, enlarged pores, inflammatory acne lesions, and overall skin tone were improved, and the patient was satisfied with the results. Pain during the nitrogen plasma and low-fluenced Nd:YAG laser treatments was tolerable without the use of topical anesthetic cream, and most of the post-treatment erythema spontaneously disappeared within 2 hours. Again, no remarkable major side effects, including itching, crusts, oozing, burn, folliculitis, prolonged edema or erythema, rebound hyperpigmentation, mottled hypopigmentation and scarring, were encountered.

\section{Case 3}

A 40-year-old Korean female visited our clinic presenting with fine, homogeneous, brownish lesions on the face. The patient had been treated with topical bleaching agents at another clinic, but the results were unsatisfactory. The lesions were symmetrically distributed primarily on the periorbital areas (Fig. 3A), and a clinical diagnosis of cluster-1 periorbital melasma was made.

After obtaining written informed consent, the patient was treated with systemic tranexamic acid $(1,000 \mathrm{mg})$ for 2 months, two sessions of fractionated ruby laser (Melastar) treatment, two sessions of IPL (ICON ${ }^{\mathrm{TM}}$ Max G Handpiece) treatment, and 13 sessions of low-fluenced 1,064nm Nd:YAG laser (RevLite ${ }^{\circledR}$ SI) treatment. We used same treatment parameters as described in cases 1 and 2 for fractionated ruby laser, IPL, and low-fluenced Nd:YAG laser treatments. While the melasma lesions improved, results were not satisfactory (Fig. 3B). Therefore, additional treatment, which comprised treatment with systemic tranexamic acid $(1,000 \mathrm{mg})$ for 2 months, 10 sessions of low-fluenced $\mathrm{Nd}$ :YAG laser treatment, and 10 sessions of nitrogen plasma treatment, were delivered. The treatment settings of the nitrogen plasma treatment included a pulse energy of $0.75 \mathrm{~J}$, a pulse duration of $10 \mathrm{msec}$, and a distance from the nozzle's tip to the skin of $10 \mathrm{~mm}$. The combined nitrogen plasma and low-fluenced Nd:YAG laser treatment and post-treatment care were performed according to methods described above.

One month after the last treatment, the patient exhibited marked reductions in melasma pigmentation along the temple, periorbital area, and anterior cheeks (Fig. 3C). Furthermore, her skin texture, enlarged pores, inflammatory acne lesions, and overall skin tone were improved. The patient was satisfied with the results. Pain during the nitrogen plasma and low-fluenced Nd:YAG laser treatments was tolerable without the use of topical anesthetic cream, and most of the post-treatment erythema spontaneously disappeared within 2 hours. No remarkable major side effects, including itching, crusts, oozing, burn, folliculitis, prolonged edema or erythema, rebound hyperpigmentation, mottled hypopigmentation and scarring, were encountered.

\section{DISCUSSION}

Plasma technology has been applied for treating a wide range of medical disorders to promote wound repair and tissue regeneration or to elicit antibacterial, antifungal, and antiviral effects. ${ }^{5-8}$ For medical purposes, various inert gaseous sources, including ambient air, argon, he-
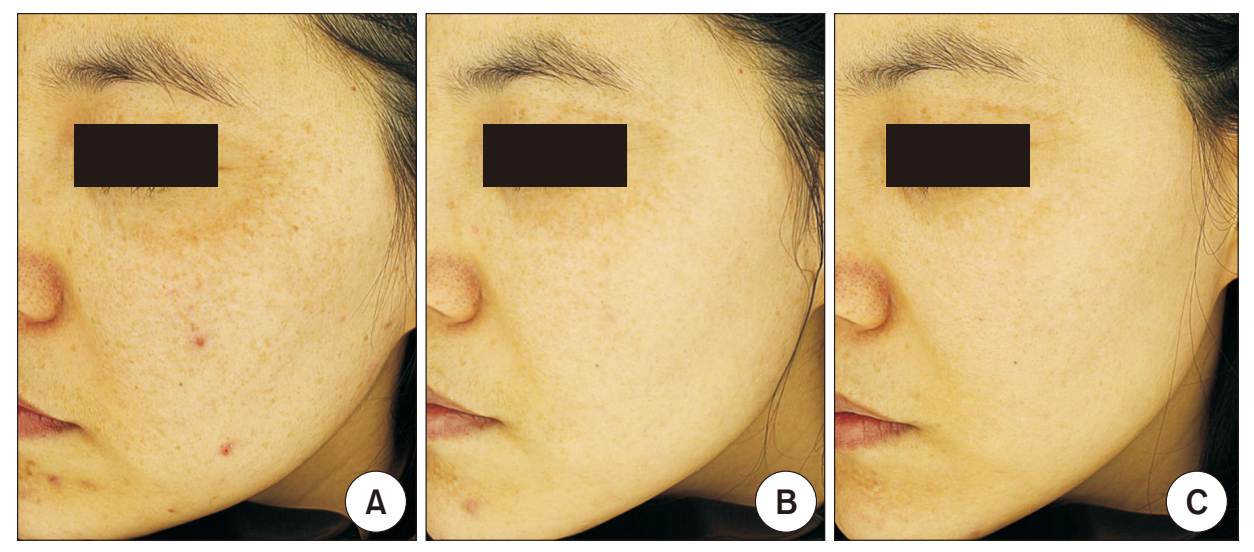

Fig. 3. Case 3. Photographs of a 40-year-old Korean female patient with cluster-2 periorbital melasma (A) at baseline; (B) 1 month after systemic tranexamic acid $(1,000 \mathrm{mg})$ treatment for 2 months, two sessions of fractionated ruby laser treatment, two sessions of IPL treatment, and 13 sessions of low-fluenced 1,064-nm Nd:YAG laser treatment; and (C) 1 month after systemic tranexamic acid (1,000 mg) treatment for 2 months, 10 sessions of low-fluenced Nd:YAG laser treatment, and 10 sessions of nitrogen plasma treatment. Photographs were taken under normal light exposure. Left lateral view. 


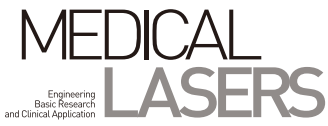

lium, and nitrogen, have been used to generate plasma excited by stripping electrons from atoms using ultrahigh frequency, radiofrequency- or microwave-based plasma generators. ${ }^{5-8}$ Our study group previously demonstrated that argon-plasma pulses generate various plasma-induced tissue reactions, ranging from microscopic vacuolar changes to thermal tissue coagulation reactions in the epidermis, follicular epithelium, and sebaceous glands. ${ }^{9}$ Meanwhile, nitrogen-plasma pulses generated energy-dependent, chromophore-independent thermal tissue coagulation and modification in the epidermis and dermis in in vivo rat skin. ${ }^{?}$

According to our previous in vivo rat study, a single pass of nitrogen plasma treatment at $0.5 \mathrm{~J}$ elicited no remarkable microscopic tissue reactions in the epidermis, with mildly dilated upper dermal microvascular components. Meanwhile, a single pass of nitrogen plasma treatment at $1.0 \mathrm{~J}$ generated mild, eosinophilic thermal tissue reactions in the epidermis, with limited vacuolar changes in basilar keratinocytes and dilated upper dermal microvascular components in the upper dermis. ${ }^{9}$ Moreover, five stacking pulses of nitrogen plasma treatment at lowenergy settings of 1.0 and $1.5 \mathrm{~J}$ generated noticeable tissue coagulation at the depths of $31.5 \pm 8.3$ and $94.9 \pm 16.9$, respectively, at Day 0.?

As described in this report, we encountered 3 patients with refractory periorbital melasma who started to present remarkable clinical improvements after adding nitrogen-plasma pulse treatment to systemic tranexamic acid and low-fluenced Nd:YAG laser treatments. The treatment settings for nitrogen plasma treatment in our study included a pulse energy of $0.75 \mathrm{~J}$, a pulse duration of $10 \mathrm{msec}$, a distance from the nozzle's tip to the skin of $10 \mathrm{~mm}$, and a single pass in all 3 patients. In accordance with our previous study, we found that a single pass of nitrogen plasma treatment at $0.75 \mathrm{~J}$ in this study could induce mild microscopic thermal tissue coagulation and modification within the epidermis by preserving the integrity of the basement membrane in patients with periorbital melasma. Moreover, we found that the nitrogen plasma pretreatment could enhance the therapeutic effects of low-fluenced Nd:YAG laser treatment without major side effects in patients with periorbital melasma.

In various pigmentation lesions, including melasma, senescent fibroblasts and endothelial cells have been suggested to secrete important melanogenic factors that significantly upregulate epidermal pigmentation. ${ }^{10}$ Therefore, therapeutic modalities for pigmentary disorders have targeted not only melanin chromophores and melanocytes in the epidermis, but also melanophages, se- nescent fibroblasts, and endothelial cells in the dermis. ${ }^{10}$ Our previous in vivo animal study demonstrated that lowenergy nitrogen plasma treatment stimulated increases in collagen fibers and fibroblasts in the upper dermis in an energy-dependent manner. ${ }^{9}$ We suggest that nitrogen plasma-induced dermal tissue regeneration could contribute to reducing senescent fibroblast-associated epidermal hyperpigmentation.

In conclusion, the cases described in this report demonstrated that nitrogen plasma treatment elicits remarkable therapeutic effects on refractory periorbital melasma lesions in Korean patients without remarkable side effects. Accordingly, we suggest that low-energy, nitrogen plasma treatment can be effectively and safely used for treating melasma lesions in addition or alternative to various treatment modalities, including systemic tranexamic acid, fractional ruby laser, IPL, and low-fluenced Nd:YAG laser treatments. Notwithstanding, further controlled investigations should be followed to elucidate the precise role of nitrogen plasma in melasma treatment.

\section{ACKNOWLEDGEMENTS}

We would like to thank Sunny Kang (Shenb Co., Ltd., Seoul, Korea), Bora Kim (Shenb Co., Ltd.), and Min Choi (Shenb Co., Ltd.) for their assistance with technical support. We would also like to thank Anthony Thomas Milliken, ELS, at Editing Synthase (https://editingsynthase. $\mathrm{com})$ for his help with the editing of this manuscript.

\section{CONFLICTS OF INTEREST}

The authors declare no conflicts of interest.

\section{REFERENCES}

1. Kang HY. Melasma and aspects of pigmentary disorders in Asians. Ann Dermatol Venereol 2012;139 Suppl 4:S1447.

2. Mandry Pagán R, Sánchez JL. Mandibular melasma. P R Health Sci J 2000;19:231-4.

3. Jung YS, Bae JM, Kim BJ, Kang JS, Cho SB. Periorbital melasma: hierarchical cluster analysis of clinical features in Asian patients. Skin Res Technol 2017;23:552-7.

4. Sheth VM, Pandya AG. Melasma: a comprehensive update: part I. J Am Acad Dermatol 2011;65:689-97.

5. Kubinova S, Zaviskova K, Uherkova L, Zablotskii V, Churpita O, Lunov O, et al. Non-thermal air plasma promotes the healing of acute skin wounds in rats. Sci Rep 2017;7:45183. 
6. Heinlin J, Zimmermann JL, Zeman F, Bunk W, Isbary $\mathrm{G}$, Landthaler M, et al. Randomized placebo-controlled human pilot study of cold atmospheric argon plasma on skin graft donor sites. Wound Repair Regen 2013;21:8007.

7. Liu JR, Xu GM, Shi XM, Zhang GJ. Low temperature plasma promoting fibroblast proliferation by activating the NF-KB pathway and increasing cyclinD1 expression. Sci Rep 2017:7:11698.

8. Cheng KY, Lin ZH, Cheng YP, Chiu HY, Yeh NL, Wu TK, et al. Wound healing in Streptozotocin-induced diabetic rats using atmospheric-pressure argon plasma jet. Sci Rep 2018:8:12214.

9. Kim H, Kim HJ, Kim HK, Hong JY, Cho SB. Effects of argon and nitrogen plasma pulses on the skin and skin appendages in an in vivo animal model. Skin Res Technol. In press 2019.

10. Kim M, Kim SM, Kwon S, Park TJ, Kang HY. Senescent fibroblasts in melasma pathophysiology. Exp Dermatol 2019:28:719-22. 